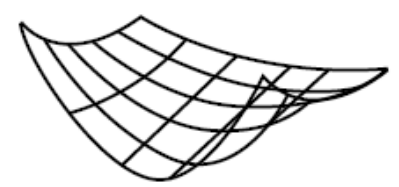

UNIVERSITY OF

TECHNOLOGY SYDNEY

QUANTITATIVE FINANCE

RESEARCH CENTRE

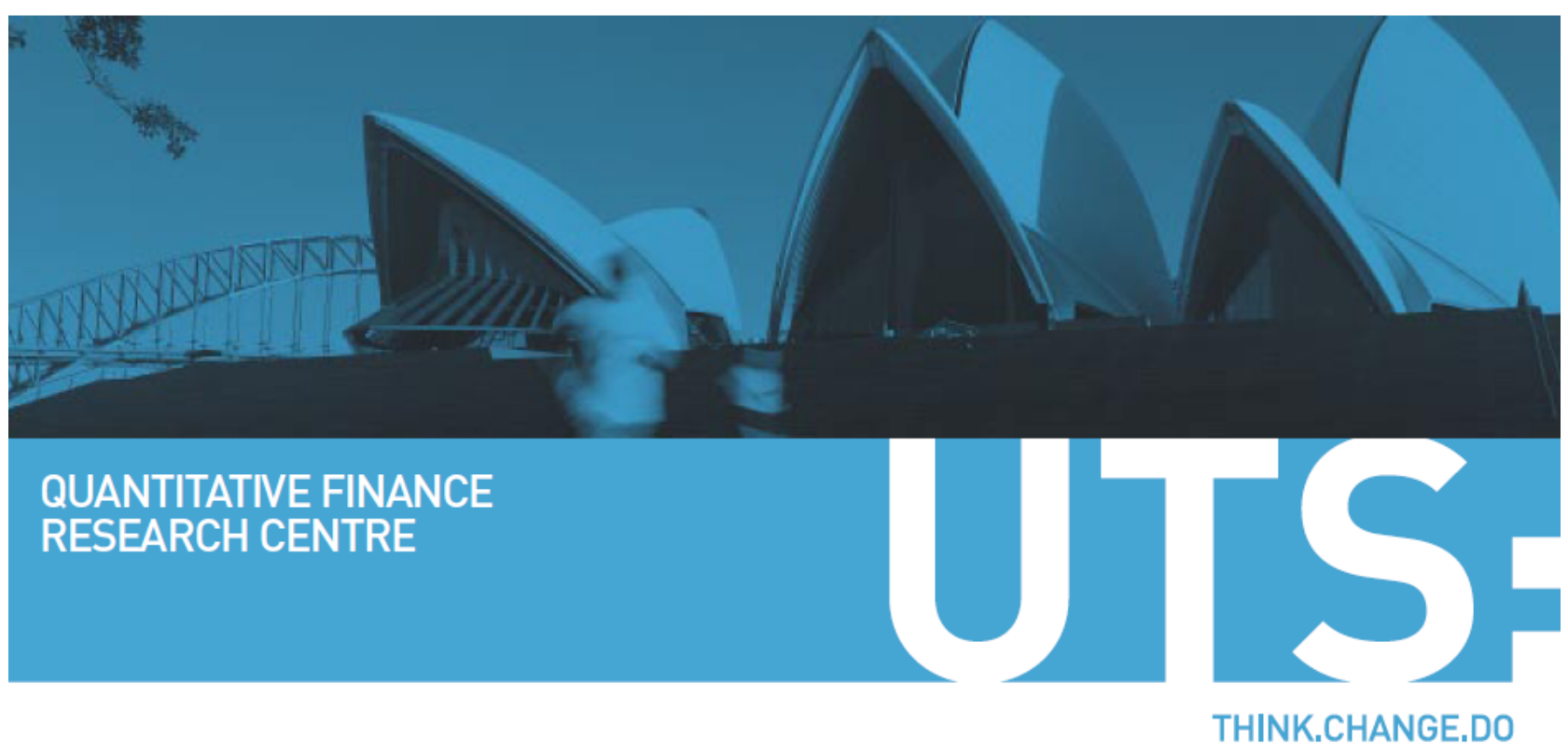

QUANTITATIVE FINANCE RESEARCH CENTRE

A Survey of Non-linear Methods for No-arbitrage

Bond Pricing

Carl Chiarella, Chih-Ying Hsiao and Ming Xi Huang 


\title{
A Survey of Non-linear Methods for No-arbitrage Bond Pricing
}

\author{
Carl Chiarella (carl.chiarella@uts.edu.au) \\ Chih-Ying Hsiao (chih-ying.hsiao@uts.edu.au) \\ Ming Xi Huang (mingxi.huang@uts.edu.au) \\ School of Finance and Economics \\ University of Technology, Sydney
}

February 9, 2010

\section{Introduction}

This survey paper studies two methods: a moment approximation method and Kimmel's method, to solve bond prices based on no-arbitrage interest models. It is well-known in the finance literature that once an interest rate model deviates from the classical affine term structure model, tractable analytical solutions for bond prices are not usually available. Approximate methods need to be adopted to give a solution for bond prices.

The first method studied here is a moment approximation method. Chiarella and Hsiao (2010) modified the moment approximation method in Takamizawa and Shoji (2009) and used it to approximate a no-arbitrage bond price as the expected discounted payoff under a risk neutral measure.

The second method is that of Kimmel (2008a, 2008b), who develops the method of time transformation to solve the partial differential equation for the pricing problem using a power series approximation. It is the case that for long time horizons pricing problem such as bond pricing, the power series approximation approach may converge slowly or not even converge at all to the exact solution. To cope with this problem, Kimmel proposes a time transformation method. With an appropriate choice of the time transformation, the power series approximation often converges for much longer time horizons.

This paper studies the behavior of the two approximate methods by apply- 
ing them to various numerical examples. All examples considered are onedimensional factor models since we will find that the application to the onedimensional examples reveals all the essential features of the approximate methods. The structure of the paper is as follows. Section 2 introduces the moment approximation method and Kimmel's method in brief. Section 3 reports the numerical results of the application of the two methods to the CIR model and a nonlinear bond model given in Goard (2000), for which she managers to obtain an analytical solution. Section 4 concludes.

\section{The Methods}

Based on modern derivative pricing theory the bond price is equal to an expected discounted future payoff

$$
P\left(t, T, x_{t}\right)=\tilde{\mathbb{E}}_{t}\left[\exp \left(-\int_{t}^{T} r_{s} d s\right)\right],
$$

with respect to some risk-neutral measure where the instantaneous rate $r_{s}$ is driven by some factor $x_{s}:=\left(x_{1 s}, \cdots, x_{n s}\right)$ and we denote $r_{s} \equiv r\left(x_{s}\right)$. The factor $x_{t}$ is an $n$-dimensional diffusion process satisfying the stochastic differential equation

$$
d x_{t}=f\left(x_{t}\right) d t+\sigma\left(x_{t}\right) d \tilde{W}_{t},
$$

where $f$ is an $\mathbb{R}^{n}$ to $\mathbb{R}^{n}$ function, $\sigma$ is an $\mathbb{R}^{n}$ to $\mathbb{R}^{n \times n}$ function and $\tilde{W}_{t}$ is an $n$-dimensional independent Wiener process under a risk-neutral measure. According to the Feynman-Kac formula the bond price $P\left(t, T, x_{t}\right)$ satisfies the partial differential equation

$$
\begin{aligned}
& \frac{\partial P}{\partial t}+\sum_{i=1}^{n} f_{i} \frac{\partial P}{\partial x_{i}}+\frac{1}{2} \sum_{i=1}^{n} \sum_{j=1}^{n} G_{i j} \frac{\partial^{2} P}{\partial x_{i} \partial x_{j}}=r\left(x_{t}\right) P, \\
& P\left(T, T, x_{T}\right)=1,
\end{aligned}
$$

where $G_{i j}$ is the $i j$-th element of the matrix $\sigma(x) \sigma(x)^{\top}$.

This paper adopts two methods to solve the no-arbitrage bond price given in Eq. (1). The first method is a moment approximation method that aims to approximate the "first moment" given in (1). The second method is proposed by Kimmel (2008a, 2008b) which approximates the solution of the PDE (3) by using power series approximations.

\subsection{The Moment Approximation Method}

Within the moment approximation method we provide two formulas. The first formula is proposed by Takamizawa and Shoji (2009). Chiarella and Hsiao 
(2010) provide a modified formula by adding a third moment term in the original formula and show that it improves accuracy of the approximation.

In order to calculate the bond price (1), we define an auxiliary variable

$$
z_{s, t}:=\exp \left(-\int_{s}^{t} r_{u} d u\right) \text {. }
$$

Using Itô's Lemma, $z_{s, t}$ follows the dynamics

$$
d z_{s, t}=-r_{t} z_{s, t} d t
$$

with the initial value $z_{s, s}=1$. Obviously, the bond pricing problem (1) becomes the problem of calculating the first moment

$$
P\left(t, T, x_{t}\right)=\tilde{\mathbb{E}}_{t}\left[z_{t, T}\right] .
$$

\subsubsection{The Takamizawa and Shoji method}

We introduce the formula of Takamizawa and Shoji (2009) for moment approximation of $r_{t}$ and $z_{t}$.

Property 1. The moment equation of the second order approximation is a 4-dimensional linear ordinary differential equation (ODE) system and is given by

$$
\Psi_{s}(t)=A(s) \int_{s}^{t} \Psi_{s}(u) d u+B(s)(t-s)+R_{s}(t)
$$

where $\Psi_{s}(t)$ is the vector of moments

$$
\Psi_{s}(t)=\left(\begin{array}{c}
\tilde{\mathbb{E}}_{s}\left[z_{s, t}-z_{s, s}\right] \\
\tilde{\mathbb{E}}_{s}\left[\left(z_{s, t}-z_{s, s}\right)\left(r_{t}-r_{s}\right)\right] \\
\tilde{\mathbb{E}}_{s}\left[r_{t}-r_{s}\right] \\
\tilde{\mathbb{E}}_{s}\left[\left(r_{t}-r_{s}\right)^{2}\right]
\end{array}\right)
$$

and

$$
A(s)=\left(\begin{array}{cccc}
-r_{s} & -1 & -1 & 0 \\
f\left(r_{s}\right) & f^{(1)}\left(r_{s}\right)-r_{s} & -r_{s} & -1 \\
0 & 0 & f^{(1)}\left(r_{s}\right) & \frac{f^{(2)}\left(r_{s}\right)}{2} \\
0 & 0 & 2 f\left(r_{s}\right)+g^{(1)}\left(r_{s}\right) & 2 f^{(1)}\left(r_{s}\right)+\frac{g^{(2)}\left(r_{s}\right)}{2}
\end{array}\right), \quad B(s)=\left(\begin{array}{c}
-r_{s} \\
0 \\
f\left(r_{s}\right) \\
g\left(r_{s}\right)
\end{array}\right) .
$$

The residual term $R_{s}(t)$ is given by

$$
R_{s}(t)=\tilde{\mathbb{E}}_{s}\left[\left(\begin{array}{c}
0 \\
-\int_{s}^{t}\left(z_{u, s}-z_{s, s}\right)\left(r_{u}-r_{s}\right)^{2} d u \\
\int_{s}^{t} \frac{1}{6} f^{(3)}\left(\xi_{1 u}\right)\left(r_{u}-r_{s}\right)^{3} d u \\
\int_{s}^{t}\left(f^{(2)}\left(\xi_{2 u}\right)+\frac{1}{6} g^{(3)}\left(\xi_{3 u}\right)\right)\left(r_{u}-r_{s}\right)^{3} d u
\end{array}\right)\right]
$$


where $\xi_{i u}$ is some value between $x_{u}$ and $x_{s}$ for every $i=1,2,3$.

Property 2. The approximate solution for $\Phi_{s}(t)$ is obtained by setting $R=0$ and is given by

$$
\Phi_{s}(t)=(A(s))^{-1}\left(e^{A(s)(t-s)}-I\right) B(s) .
$$

According to Shoji (2002), if we have global differentiablity of the functions $f$ and $g$ we can control the error by use of the result that

$$
\Psi_{s}(t)-\Phi_{s}(t)=\mathcal{O}\left((t-s)^{\frac{n+3}{2}}\right) .
$$

Note From the error control (9) we do not know whether the approximation $\Phi_{s}(t)$ is close to the real solution $\Psi_{s}(t)$ as the time difference $t-s$ becomes large. In the bond pricing formula, this time difference is the time to maturity of a bond, which can reach twenty years for a standard long term bond. In order to reduce the error $\Psi_{s}(t)-\Phi_{s}(t)$, if we cannot cut the time difference $t-s$, we include more terms in the error (7), which gives rise to the following modified formula for the moment approximation method.

\subsubsection{A modified formula}

In the following modified formula, we include the term $\tilde{\mathbb{E}}_{s}\left[\left(z_{s, u}-z_{s, s}\right)\left(r_{u}-r_{s}\right)^{2}\right]$ into the moment dynamics.

Property 3. The modified moment equation of the second order approximation is a five dimensional ODE system and is given by

$$
\Psi_{s}^{a d}(t)=A_{s}^{a d} \int_{s}^{t} \Psi_{s}^{a d}(u) d u+B_{s}^{a d}(t-s)+R_{s}^{a d}(t)
$$

where we define the moments

$$
\Psi_{s}^{a d}(t)=\left(\begin{array}{c}
\tilde{\mathbb{E}}_{s}\left[z_{s, t}-z_{s, s}\right] \\
\tilde{\mathbb{E}}_{s}\left[\left(z_{s, t}-z_{s, s}\right)\left(r_{t}-r_{s}\right)\right] \\
\tilde{\mathbb{E}}_{s}\left[r_{t}-r_{s}\right] \\
\tilde{\mathbb{E}}_{s}\left[\left(r_{t}-r_{s}\right)^{2}\right] \\
\tilde{\mathbb{E}}_{s}\left[\left(z_{s, t}-z_{s, s}\right)\left(r_{t}-r_{s}\right)^{2}\right]
\end{array}\right)
$$

and

$$
A_{s}^{a d}=\left(\begin{array}{ccccc}
-r_{s} & -1 & -1 & 0 & 0 \\
f\left(r_{s}\right) & f^{(1)}\left(r_{s}\right)-r_{s} & -r_{s} & -1 & -1+\frac{f^{(2)}\left(r_{s}\right)}{2} \\
0 & 0 & f^{(1)}\left(r_{s}\right) & \frac{f^{(2)}\left(r_{s}\right)}{2} & 0 \\
0 & 0 & 2 f(s)+g^{(1)}\left(r_{s}\right) & 2 f^{(1)}\left(r_{s}\right)+\frac{g^{(2)}\left(r_{s}\right)}{2} & 0 \\
g\left(r_{s}\right) & 2 f\left(r_{s}\right)+g^{(1)}\left(r_{s}\right) & 0 & -r_{s} & 2 f^{(1)}\left(r_{s}\right)+\frac{g^{(2)}\left(r_{s}\right)}{2}-r_{s}
\end{array}\right),
$$




$$
B_{s}^{a d}=\left(\begin{array}{c}
-r_{s} \\
0 \\
f\left(r_{s}\right) \\
g\left(r_{s}\right) \\
0
\end{array}\right)
$$

The error term now becomes

$$
R_{s}^{a d}(t)=\tilde{\mathbb{E}}_{s}\left[\left(\begin{array}{c}
0 \\
\int_{s}^{t} \frac{f^{(3)}\left(\xi_{4 u}\right)}{6}\left(z_{s, u}-z_{s, s}\right)\left(r_{u}-r_{s}\right)^{3} d u \\
\int_{s}^{t} \frac{f^{(3)}\left(\xi_{1 u}\right)}{6}\left(r_{u}-r_{s}\right)^{3} d u \\
\int_{s}^{t}\left(f^{(2)}\left(\xi_{2 u}\right)+\frac{g^{(3)}\left(\xi_{3 u}\right)}{6}\right)\left(r_{u}-r_{s}\right)^{3} d u \\
\int_{s}^{t}\left(6 f^{(2)}\left(\xi_{5 u}\right)+g^{(3)}\left(\xi_{6 u}\right)-z_{s, u}\right)\left(r_{u}-r_{s}\right)^{3} d u
\end{array}\right)\right],
$$

where $\xi_{i u}$ are values between $r_{u}$ and $r_{s}$ for all $i=1, \cdots, 6$.

Similarly, the approximate solution is given by the next property.

Property 4. Let $\Phi_{s}^{a d}(t)$ be the approximate solution satisfying

$$
\Phi_{s}^{a d}(t)=A_{s}^{a d} \int_{s}^{t} \Psi_{s}^{a d}(u) d u+B_{s}^{a d} \cdot(t-s) .
$$

Then $\Phi_{s}^{a d}(t)$ is given by

$$
\Phi_{s}^{a d}(t)=\left(A_{s}^{a d}\right)^{-1}\left(e^{A_{s}^{a d} \cdot(t-s)}-I_{5}\right) B_{s}^{a d} .
$$

The errors between the original process $\Psi_{s}^{a d}(t)$ and the approximate solution $\Phi_{s}^{a d}(t)$ can be bounded by a simple process.

Property 5. Assume that the error term (11) can be bounded by the linear equation

$$
R_{s}^{a d}(t) \leq R_{\max } \cdot(t-s) .
$$

Then the approximation error $\left|\Psi_{s}^{a d}(t)-\Phi_{s}^{a d}(t)\right|$ can be controlled by

$$
\left|\Psi_{s}^{a d}(t)-\Phi_{s}^{a d}(t)\right| \leq\left(A_{s}^{a d}\right)^{-1}\left(e^{A_{s}^{a d} \cdot(t-s)}-I_{5}\right) R_{\max } .
$$

Proof see Appendix.

\section{$2.2 \quad$ Kimmel's Method}

An alternative method is developed in Kimmel (2008b) (hereafter Kimmel's method) to solve the $P$ satisfying the PDE (3). Kimmel (2008b) suggests the 
solution of the PDE (3) with the final payoff $\phi(x)$ can be approximated by applying the power series in $\tau=T-t$ time-to-maturity, centred at zero,

$$
P(\tau, x)=a_{0}(x)+\sum_{n=1}^{\infty} a_{n}(x) \frac{\tau^{n}}{n !},
$$

where $a_{0}(x)$ and $a_{n}(x)$ are recursively related coefficients of up to a number $N$ of terms:

$$
\begin{aligned}
& a_{0}(x)=\phi(x) \\
& a_{n}(x)=\sum_{i=1}^{N} f_{i} \frac{\partial a_{n-1}}{\partial x_{i}}+\frac{1}{2} \sum_{i=1}^{N} \sum_{j=1}^{N} G_{i j} \frac{\partial^{2} a_{n-1}}{\partial x_{i} \partial x_{j}}-r(x) a_{n-1} .
\end{aligned}
$$

Note that for notational convenience, here we drop the subscript $t$ on $x$ in (3).

Kimmel (2008b) explains that it is difficult to determine whether a given $\phi(x)$ lies within the class that generate solutions with convergent power series. He therefore illustrates two families of scalar PDEs for which it is possible to have solutions via convergent power series in the one-dimensional situation. The two families of scalar PDEs are

$$
\begin{aligned}
\frac{\partial h(\tau, y)}{\partial \tau} & =\frac{1}{2} \frac{\partial^{2} h}{\partial y^{2}}-\beta(y) h, \\
h(0, y) & =g(y),
\end{aligned}
$$

where the two specifications of $\beta(y)$ are

$$
\begin{aligned}
& \beta(y)=\frac{b^{2}}{2}(y-a)^{2}+d, \\
& \beta(y)=\frac{a}{y^{2}}+\frac{b^{2}}{2} y^{2}+d,
\end{aligned}
$$

where $a, b$ and $d$ are constants. The transformation of the pricing function $P(\tau, x)$ to the function $h(\tau, y)$ is based on the change of variable techniques of Colton (1979). More details are shown in Kimmel (2008a).

However, Kimmel points out that in the applications for large values of $\tau$, the convergence of the power series presents some difficulties. For example, the limitation of convergence for positive $\tau$, or very slow convergence for large values of $\tau$. In order to deal with the large values of $\tau$, Kimmel (2008b) introduces a "changing time" method, which transforms the time-frame to an exponential function, namely

$$
\xi \equiv 1-\exp (-\gamma \tau),
$$

where $\gamma \neq 0$ and it is most useful to choose $\gamma$ to be a real positive number in typical applications. 
In next section, we show how the "changing times" method works by illustrating the CIR example in Kimmel (2008b), and compare the results to the moment approximation method developed in Subsection 2.1. We also investigate the possibility of extending the "changing times" method to the general case, and study the limitations of this approach.

\section{Testing of the Methods with Examples}

This section tests the numerical performance of the two nonlinear methods for solving no-arbitrage bond price. The first example is the CIR interest rate model in Cox, Ingersoll and Ross (1985)?. The second example is a non-linear interest rate model studied by Goard (2000).

\subsection{Application of the moment approximation method to the CIR model}

We apply the two formulas of the moment approximation method to a CIR interest rate model. In the CIR model, the risk neutral interest rate dynamics are given by

$$
d r_{t}=\kappa\left(\theta-r_{t}\right) d t+\sigma \sqrt{r_{t}} d \tilde{W}_{t},
$$

where $\tilde{W}_{t}$ is the Brownian motion under the risk-neutral measure.

For the simulation study we choose $\kappa=0.5, \theta=0.08$ and $\sigma=0.15$. The panels of Figure 4 illustrate the relative errors of the bond prices based on the modified formula (13) where the first panel depicts the relative errors for different interest rates $r_{t}$ and time to maturities $\tau$. The errors are small for short times to maturity and increase as maturity becomes longer. Looking more in detail in the second panel different curves from bottom to top corresponds to different time spans to maturity from one year to ten years. The average errors remain small around the equilibrium level $\theta=0.08$ for all maturities but increase by a great amount at the two ends far from the equilibrium. In the third panel the shaded area gives the $\left(r_{t}, \tau\right)$ combination at which the relative error is controlled within $1 \%$. The error is larger than $1 \%$ only at extremely high interest rate levels with maturity longer than 9 years.

For error analysis we refer to the residual term (11), where the higher order derivatives $f^{(2)}, f^{(3)}$ and $g^{(3)}$ are all equal to zero. The only nonzero term in the residual is $-\int_{s}^{t} z_{s, u}\left(r_{u}-r_{s}\right)^{3} d u$. The residual becomes $-\int_{t}^{T} z_{t, u}\left(r_{u}-r_{t}\right)^{3} d u$ for the calculation of the bond price $P\left(t, T, r_{t}\right)$. Since the CIR process is stationary $r_{u}$ for $u \in[t, T]$ will approach and fluctuate around the equilibrium level $\theta$. 
An initial rate $r_{t}$ far from the equilibrium level will induce a big gap in $\left(r_{u}-r_{s}\right)^{3}$ and this gap accumulates over the time interval $[t, T]$. Relating this to the error control upper bound given in (14) we see that the large deviation of $r_{t}$ from the equilibrium level induces a large value of $R_{\max }$. Applying this knowledge to the error control equation (15) we can explain two features concerning the errors observed from our results in Figure 4.

- In the second panel we see the longer the time to maturity $T-t$ the bigger are the errors. This can be explained by the fact that the upper bound of the error $\left(A_{t}^{a d}\right)^{-1}\left(e^{A_{t}^{a d} \cdot(T-t)}-I_{5}\right) R_{\max }$ in (15) increases with time to maturity $T-t$.

- For each curve in the second panel (corresponding to a fixed time to maturity) the more the interest rate moves from the equilibrium level, the bigger the errors. This is because $R_{\max }$ in (15) increases as the initial rate $r_{t}$ moves away from the equilibrium.

In order to compare with a nonlinear example later we depict the relative errors for a higher mean reversion speed $\kappa=2.5$ in the panels of Figure 3 . We find that (1) the errors show the two features as analyzed above. (2) The errors of the moment method reduce for a higher $\kappa$. The biggest error is still less than $0.15 \%$ as shown in the second panel.

Table 3 summarizes the numerical performance of the approximate scheme of Takamizawa and Shoji (2009) and Chiarela and Hsiao (2010) together with Kimmel's method introduced in detail in the next Section.

\subsection{Application of Kimmel's Method to the CIR Model}

In this subsection, we focus on the application of Kimmels method to solve the CIR model. Subsection 3.2.1 shows that how Kimmel's special transformation (including the time and state transformation) approach works by illustrating the CIR example in Kimmel (2008b). In subsection 3.2.2, we solve the CIR model using only time transformation approach. In subsection 3.2.3, we investigate the possibility of extending the "changing times" method to the general case.

\subsubsection{Kimmel's special transformation approach}

In this subsection we illustrate Kimmel's special transformation approach given in Kimmel (2008b) to solve the CIR model ${ }^{1}$. Consider the CIR model in (24),

\footnotetext{
${ }^{1}$ See the second example in Kimmel (2008b).
} 
for which the PDE (3) becomes

$$
\begin{aligned}
\frac{\partial P}{\partial \tau} & =\frac{1}{2} \sigma^{2} r \frac{\partial^{2} P}{\partial r^{2}}+\kappa(\theta-r) \frac{\partial P}{\partial r}-r P, \\
P(0, r) & =1 .
\end{aligned}
$$

Kimmel (2008b) shows that the pricing function (25) can be solved by a convergent power series approximation after a special time and state transformation. The first step is to transform the pricing function (25) to the scalar PDE as described in (19), namely

$$
y(r)=\frac{2 \sqrt{r}}{\sigma},
$$

and make the transformation

$$
P(\tau, r)=\left(\frac{4 r}{\sigma^{2}}\right)^{\frac{1}{4}-\frac{\theta \kappa}{\sigma^{2}}} e^{\kappa r / \sigma^{2}} h(\tau, y(r)) .
$$

The function $h(\tau, y(r))$ satisfies the PDE

$$
\frac{\partial h(\tau, y)}{\partial \tau}=\frac{1}{2} \frac{\partial^{2} h}{\partial y^{2}}-\beta(y) h,
$$

with the initial condition

$$
h(0, y)=y^{\alpha} e^{-\kappa y^{2} / 4},
$$

where

$$
\beta(y) \equiv\left[\frac{a}{y^{2}}+\frac{b^{2}}{2} y^{2}+d\right],
$$

and

$$
\begin{aligned}
a & \equiv-\frac{2 \theta^{2} \kappa^{2}}{\sigma^{4}}-\frac{2 \theta \kappa}{\sigma^{2}}+\frac{3}{8} \\
b & \equiv \frac{\sqrt{\kappa^{2}+2 \sigma^{2}}}{2} \\
d & \equiv \frac{\kappa^{2} \theta}{\sigma^{2}} \\
\alpha & \equiv \frac{1+\sqrt{1+8 a}}{2}
\end{aligned}
$$

Next, taking Kimmel's special time and state transformation,

$$
\xi=1-e^{-2 b \tau},
$$


choosing $\gamma=2 b$ and setting

$$
z=\sqrt{2 b} e^{-b \tau} y
$$

the PDE (29) transforms to

$$
h(\tau, y) \equiv e^{-\frac{b}{2} y^{2}-\left(\frac{b}{2}+d\right) \tau}\left(\frac{z}{\sqrt{2 b}}\right)^{\frac{1+\sqrt{1+8 a}}{2}} w(\xi, z),
$$

where the function $w(\xi, z)$ satisfies $^{2}$

$$
\begin{aligned}
\frac{\partial w(\xi, z)}{\partial \xi} & =\frac{1}{2} \frac{\partial^{2} w}{\partial z^{2}}+\frac{1+\sqrt{1+8 a}}{2 z} \frac{\partial w}{\partial z} \\
w(0, z) & =e^{z^{2}(0.25-0.125 \kappa / b)} .
\end{aligned}
$$

The final step is to apply a power series approximation to equation (39) in $\xi$, so that

$$
w(\xi, z)=b_{0}(z)+\sum_{n=1}^{N} b_{n}(z) \frac{\xi^{n}}{n !}
$$

where

$$
b_{0}(z)=w(0, z)
$$

and

$$
b_{n}(z)=\frac{1}{2} b_{n-1}^{\prime \prime}(z)+\frac{1+\sqrt{1+8 a}}{2 z} b_{n-1}^{\prime}(z),
$$

where

$$
b_{n}^{\prime}(z)=\frac{\partial}{\partial z} b_{n}(z) \quad \text { and } \quad b_{n}^{\prime \prime}(z)=\frac{\partial^{2}}{\partial z^{2}} b_{n}(z) .
$$

\subsubsection{Approach with time transformation only}

In the previous subsection, the CIR example in Kimmel (2008b) shows how the power series approach works when applying Kimmel's special transformation. In this subsection, in order to isolate the effect between time and state transformation, we apply the time transformation only to solve the CIR model, namely

$$
h(\tau, y)=u(\xi, y),
$$

where $\xi$ is expressed in (23).

\footnotetext{
${ }^{2}$ See Kimmel (2008b) Theorem 5 for the proof and discussion.
} 
Substituting (44) into (29)-(30), the PDE becomes

$$
\gamma(1-\xi) \frac{\partial u(\xi, x)}{\partial \xi}=\frac{1}{2} \frac{\partial^{2} u}{\partial y}-\beta(y) u,
$$

with initial condition

$$
u(0, y)=y^{\alpha} e^{-\kappa y^{2} / 4} .
$$

The power series representation of $u(\xi, y)$ in $\xi$ is

$$
u(\xi, y)=c_{0}(y)+\sum_{n=1}^{\infty} c_{n}(y) \frac{\xi^{n}}{n !},
$$

where $c_{0}(x)$ and $c_{n}(x)$ are recursively generated coefficients given by

$$
\begin{aligned}
c_{0}(y) & =u(0, y) \\
c_{n}(y) & =\frac{1}{4 b} c_{n-1}^{\prime \prime}-\left[\frac{\beta(y)}{2 b}+1-n\right] c_{n-1} .
\end{aligned}
$$

\subsubsection{Extending Kimmel's method by the second order approxima- tion approach}

According to Kimmel (2008b), the "changing time" approach can only be applied for certain special forms of the pricing problems, such as Ahn, Dittmar and Gallant (2002) (ADG). In this subsection, we seek to extend the Kimmel's method to a general case by applying a second order approximation approach. We attempt this by using a simple case, which is to apply the second order approach to approximate CIR model using the ADG model with Kimmel's special transformation framework.

The ADG model is give by

$$
\begin{aligned}
d x_{t} & =\kappa\left(\theta-x_{t}\right) d t+\sigma d \tilde{W}_{t} \\
r_{t} & =x_{t}^{2}+\phi
\end{aligned}
$$

and the zero-coupon bond prices $P(\tau, x)$ satisfies the PDE

$$
\frac{\partial P}{\partial \tau}=\frac{1}{2} \sigma^{2} \frac{\partial^{2} P}{\partial x^{2}}+\kappa(\theta-x) \frac{\partial P}{\partial x}-\left(x^{2}+\phi\right) P,
$$

with initial condition

$$
P(0, x)=1
$$


where $\tau$ is the time-to-maturity.

Kimmel shows that there are three steps for solving the PDE (52) by the method of the time transformation.

Step 1: Perform change of variables

$$
\hat{y}(x)=\frac{x-\phi}{\sigma},
$$

and carry out the transformation from $P(\tau, x)$ to $h(\tau, \hat{y}(x))$ via

$$
P(\tau, x)=e^{\frac{\kappa(\theta-x)^{2}}{2 \sigma^{2}}} h(\tau, \hat{y}(x)) .
$$

The function $h(\tau, \hat{y}(x))$ satisfies the PDE

$$
\frac{\partial h(\tau, \hat{y})}{\partial \tau}=\frac{1}{2} \frac{\partial^{2} h(\tau, \hat{y})}{\partial \hat{y}^{2}}-\hat{\beta}(\hat{y}) h(\tau, \hat{y}),
$$

with initial condition

$$
h(0, \hat{y})=e^{-\kappa \hat{y}^{2} / 2},
$$

where

$$
\hat{\beta}(\hat{y}) \equiv\left[\frac{\hat{b}^{2}}{2}(\hat{y}-\hat{a})^{2}+\hat{d}\right],
$$

and

$$
\begin{aligned}
\hat{a} & \equiv-\frac{2 \theta \sigma}{\hat{b}^{2}}, \\
\hat{b} & \equiv \sqrt{\kappa^{2}+2 \sigma^{2}}, \\
\hat{d} & \equiv \frac{\kappa^{2} \theta^{2}}{\hat{b}^{2}}-\frac{\kappa}{2}+\phi .
\end{aligned}
$$

Step 2: Apply the special time and state transformation

$$
\hat{\xi}=1-e^{-2 \hat{b} \tau},
$$

and

$$
\hat{z}=\sqrt{2 \hat{b}}\left[\hat{a}+e^{-\hat{b} \tau}(\hat{y}-\hat{a})\right] .
$$

Then transform from $h(\tau, \hat{y})$ to $w(\hat{\xi}, \hat{z})$ according to

$$
h(\tau, \hat{y}) \equiv e^{-\frac{\hat{b}}{2}(\hat{y}-\hat{a})^{2}-\left(\frac{\hat{b}}{2}+\hat{d}\right) \tau} w(\hat{\xi}, \hat{z}),
$$


to reduce the PDE to $w(\hat{\xi}, \hat{z})$ which satisfies

$$
\frac{\partial w(\hat{\xi}, \hat{z})}{\partial \hat{\xi}}=\frac{1}{2} \frac{\partial^{2} \hat{w}}{\partial \hat{z}^{2}}
$$

with initial condition

$$
w(0, \hat{z})=e^{\frac{1}{4}(\hat{z}-\hat{a} \sqrt{2 \hat{b}})^{2}} \exp \left(-\frac{\kappa \hat{z}^{2}}{4 \hat{b}}\right),
$$

The proof can be found in Kimmel (2008b) Theorem 4 .

Step 3: Apply the power series approximation for $(65)$ in $\hat{\xi}$ :

$$
w(\hat{\xi}, \hat{z})=b_{0}(\hat{z})+\sum_{n=1}^{N} b_{n}(\hat{z}) \frac{\hat{\xi}^{n}}{n !},
$$

where

$$
b_{0}(\hat{z})=w(0, \hat{z})
$$

and

$$
b_{n}(\hat{z})=\frac{1}{2} b_{n-1}^{\prime \prime}(\hat{z}) .
$$

Based on the ADG framework illustrated above, we apply a Taylor expansion to (31) around $y_{0}$ up to the second-order term, hence we have

$$
\beta(y)=\beta\left(y_{0}\right)+\left(y-y_{0}\right) \beta^{\prime}\left(y_{0}\right)+\left(y-y_{0}\right)^{2} \beta^{\prime \prime}\left(y_{0}\right),
$$

where $\beta^{\prime}\left(y_{0}\right)$ and $\beta^{\prime \prime}\left(y_{0}\right)$ are the first and second derivatives with respect to $y_{0}$. The second approximation depends on the choice of $y_{0}$ as shown in Figure 3.2.3 for the CIR example.

Completing the square, we have

$$
\beta(y)=\frac{1}{2} \beta^{\prime \prime}\left(y_{0}\right)\left[y-\left(y_{0}-\frac{\beta^{\prime}\left(y_{0}\right)}{\beta^{\prime \prime}\left(y_{0}\right)}\right)\right]-\frac{1}{2} \frac{\left[\beta^{\prime}\left(y_{0}\right)\right]^{2}}{\beta^{\prime \prime}\left(y_{0}\right)}+\beta\left(y_{0}\right) .
$$

Next, comparing equation (71) to equation (58), we can rewrite (58)-(61) as

$$
\hat{\beta}(y) \equiv\left[\frac{\hat{b}^{2}}{2}(y-\hat{a})^{2}+\hat{d}\right],
$$

where

$$
\begin{aligned}
\hat{b} & \equiv \sqrt{\beta^{\prime \prime}\left(y_{0}\right)}, \\
\hat{a} & \equiv y-\frac{\beta^{\prime}\left(y_{0}\right)}{\beta^{\prime \prime}\left(y_{0}\right)}, \\
\hat{d} & \equiv \beta\left(y_{0}\right)-\frac{\left[\beta^{\prime}\left(y_{0}\right)\right]^{2}}{\beta^{\prime \prime}\left(y_{0}\right)} .
\end{aligned}
$$


Finally, the CIR model is approximated by the ADG model framework (Steps 1-3) by replacing (58)-(61) by (72)-(75) and $\hat{y}$ by $y$.

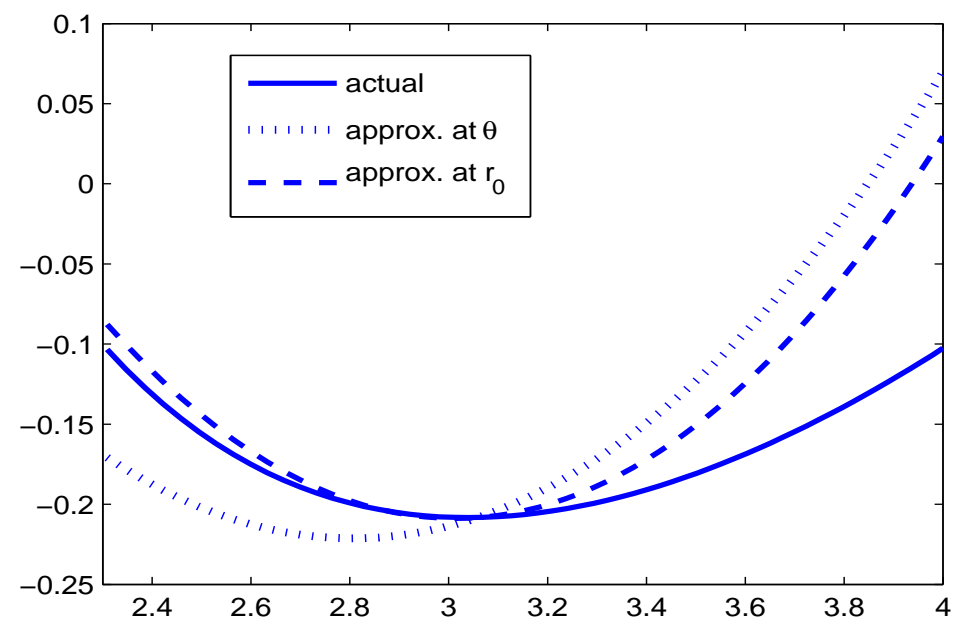

Figure 1: The plot for the actual function of $\bar{\beta}(\bar{y})$ in (31) and its second-order approximation function in (71). The solid line corresponds to the actual function. The dotted line corresponds to the approximation function using $\bar{y}_{0}=\frac{2 \sqrt{\theta}}{\sigma}$, and the dashed line corresponds to the approximation function using $\bar{y}_{0}=\frac{2 \sqrt{r_{0}}}{\sigma}$.

\subsection{Summary of the Numerical Results}

Table 3 summarizes the numerical results based on the moment approximation method and the Kimmel's method applied to the CIR model. Comparing the two formulas within the moment approximation method the modified formula (CH) by Chiarella and Hsiao (2010) provides significant improvement over the original method of Takamizawa and Shoji (2009) in the bond price calculation.

Regarding Kimmel's method we compare the three procedures based on subsection 3.2.1, the approach with time transformation only in subsection 3.2.2, and the second order approximation approach developed in subsection 3.2.3. Table 3 summarizes results of those procedures for power series up to order four. It shows that the results of Kimmel's special transformation and the approach using only the time transformation are both smaller than $1 \%$ relative percentage error for bond price and yield when $T=1$. For the second order approximation approach, the relative percentage error of bond price and yield when $T=1$ are less than $1 \%$ and around $7 \%$ respectively. However, as $T$ increases to $T=5$ and $T=10$, the relative percentage error for both bond prices and yields based 
on only the time transformation method and the second order approximation method are larger than $10 \%$. While, the relative percentage error of bond price and yield using Kimmel's special transformation method are all less than $1 \%$ for $T=1,5$ and 10. It shows the fact that use of the power series approximation based on Kimmel's special transformation provides smaller error, while based on only the time transformation and the second order approximation methods, the error are very large when $T$ is 5 or 10 years. In other words, the numerical results also show that Kimmel's method only works well for some specific transformations, which are applicable only to certain bond pricing models. The second-order approach developed in Subsection 3.2.3 attempts to generalize the changing times approach but does not provide an accurate approximation.

Comparing the moment approximation method and Kimmel's method, the moment approximation method outperforms even the special transformation $w$ in Kimmel's method for $T=1$, equally well for $T=5$ but slightly worse for longer maturity $T=10$.

\subsection{An Example from Goard (2000)}

In this subsection, we try an example from Goard (2000) which has a closedform solution that can serve as a check. We choose the first example in Goard (2000), where the interest rate follows the risk-neutral process as

$$
d r_{t}=f(r) d t+\sigma(r) d \tilde{W}_{t}
$$

where

$$
\begin{aligned}
& f(r)=\left[c^{2} r_{t}\left(\delta-q r_{t}\right)\right], \\
& \sigma(r)=c r_{t}^{3 / 2} .
\end{aligned}
$$

Note, in Goard (2000), $\delta$ can be time varying, but for simplicity we consider $\delta$ to be constant here.

\subsubsection{Application of the moment approximation method}

We apply the the moment approximation method (13) to the example (76). We rewrite the drift coefficient as a mean reverting term

$$
f(r)=c^{2} q r_{t}\left(\theta-r_{t}\right), \quad \theta:=\frac{\delta}{q}
$$

where the process has a level-dependent reversion speed $c^{2} q r_{t}$ and an equilibrium level $\theta$.

The panels in Figure 5 provide descriptions of the relative errors of the application for the case $c=1.0, \delta=2.4$ and $q=30$. The equilibrium level in this case 
is $\theta=8 \%$. The impact of the nonlinearity given in the dynamics (76) on the performance of the moment approximation method can be seen by comparing to our previous CIR example shown in Figure 3. The parameters in the drift term are comparable to fixing $r_{t}$ in (76) at $\theta$ as they summarized in Table 1.

\begin{tabular}{c|ccc} 
& mean reversion & equilibrium & volatility \\
\hline CIR (24) Fig. 3 & $\kappa=2.5$ & $\theta=0.08$ & $\sigma=0.15$ \\
Goard (76) Fig. 5 & $c^{2} q \theta=2.4$ & $\theta=0.08$ & $c \theta^{1.5}=0.0226$
\end{tabular}

Table 1: Parameter Comparison

On the one hand, the errors show similar pattern as for the CIR model in Section 3.1, namely, (a) the errors increase as time to maturity increases; (b) the errors are small around the equilibrium level for all times to maturity while they increase by a larger amount with time to maturity as the interest rate moves far from the equilibrium. On the other hand, the errors behave differently than in the CIR example where the errors are much bigger than the CIR case shown in Figure 3. This can be explained by the residual term given in eq. (11). For the CIR model all higher order derivative terms are equal to zero while for the case $(76)$ we have $f^{(3)}(\xi)=0$ but $f^{(2)}(\xi)=2 c^{2} a q$ and $g^{(3)}(\xi)=6 c^{2}$ so those will result larger residuals than those in the CIR model.

Figure 6 provides the results of relative errors for a slow mean reversion speed with $q=6$. The impact of nonlinearity is shown by comparing to results of the CIR model given in Figure 4. Their drift parameters are comparable as can be seen in Table 2.

\begin{tabular}{c|ccc} 
& mean reversion & equilibrium & volatility \\
\hline CIR (24) Fig. 4 & $\kappa=0.5$ & $\theta=0.08$ & $\sigma=0.15$ \\
Goard (76) Fig. 6 & $c^{2} q \theta=0.48$ & $\theta=0.08$ & $c \theta^{1.5}=0.0226$
\end{tabular}.

Table 2: Parameter Comparison (slower mean reversion)

The errors have again the same features of increasing with time to maturity and are U-shaped in the direction of the initial interest rate. Also the nonlinearity increases the error of the moment approximation. However, we still observe one point of different. In the CIR model, the moment approximation method performs better for a higher mean reversion speed. While this is not the case for Goard's nonlinear example. 


\subsubsection{Application of Kimmel's method}

The bond prices $P(\tau, r)$ based on the interest rate dynamics (76) satisfies the PDE

$$
\frac{\partial P}{\partial \tau}=\frac{1}{2} c^{2} r^{3} \frac{\partial^{2} P}{\partial r^{2}}+\left[c^{2} r(\delta-q r)\right] \frac{\partial P}{\partial r}-r P,
$$

with initial condition

$$
P(0, r)=1
$$

In order to apply Kimmel's method, the first derivative in the partial differential equation (80) has to be eliminated. Kimmel (2008a) describes the approach of Colton (1979) for elimination of the first spatial derivative term from the PDE as follows (see equations (4.2)-(4.5) in Kimmel, Jan2008)

$$
\begin{aligned}
y & =\int^{r} \frac{1}{\sigma(u)} d u \\
P(\tau, r) & =\exp \left(-\int^{r}\left[\frac{\mu(u)}{\sigma^{2}(u)}-\frac{\sigma^{\prime}(u)}{2 \sigma(u)}\right] d u\right) h(\tau, y) .
\end{aligned}
$$

Noted that as pointed out in Kimmel (2008a) the lower limits of the integrals are not specified, so these expressions really describe a family of transforms. He also states that positivity and continuity of $\sigma(r)$ on the interior of the state space means ensure that $y$ is a strictly increasing function of $r$, and therefore can be inverted.

The transformed differential equation $h(\tau, y)$ then satisfies

$$
\frac{\partial h}{\partial \tau}=\frac{1}{2} \frac{\partial^{2} h}{\partial y^{2}}-\beta(y) h
$$

where

$$
\beta(y) \equiv-\frac{\mu^{2}(r)}{2 \sigma^{2}(r)}-\frac{\mu^{\prime}(r)}{2}+\frac{\mu(r) \sigma^{\prime}(r)}{\sigma(r)}-\frac{\mu^{\prime}(r) \mu^{\prime}(r)}{8}-\frac{\sigma^{\prime \prime}(r) \sigma(r)}{4}-r .
$$

Applying the above procedures (82)-(85) to the pricing function (80), we have

$$
\begin{aligned}
y & =-\frac{2}{c \sqrt{r}}, \\
P(\tau, r) & =e^{\delta / r} r^{3 / 4+q} h(\tau, y),
\end{aligned}
$$

where $h(\tau, y)$ satisfies (84) and

$$
\beta(y)=\frac{-3 / 8-4 / c^{2}-2 q-2 q^{2}}{y^{2}}-\frac{1}{8} \delta^{2} c^{4} y^{2}+\delta c^{2}(1+q) .
$$


The result for $\beta(y)$ in (88) prevents us from applying Kimmel's special transformation procedure, which starts with the functional form of $\beta(y)$ given in (31). The coefficient of $y^{2}$ in (88) is negative while the corresponding coefficient in (31) is $\frac{b^{2}}{2}$, which is always positive.

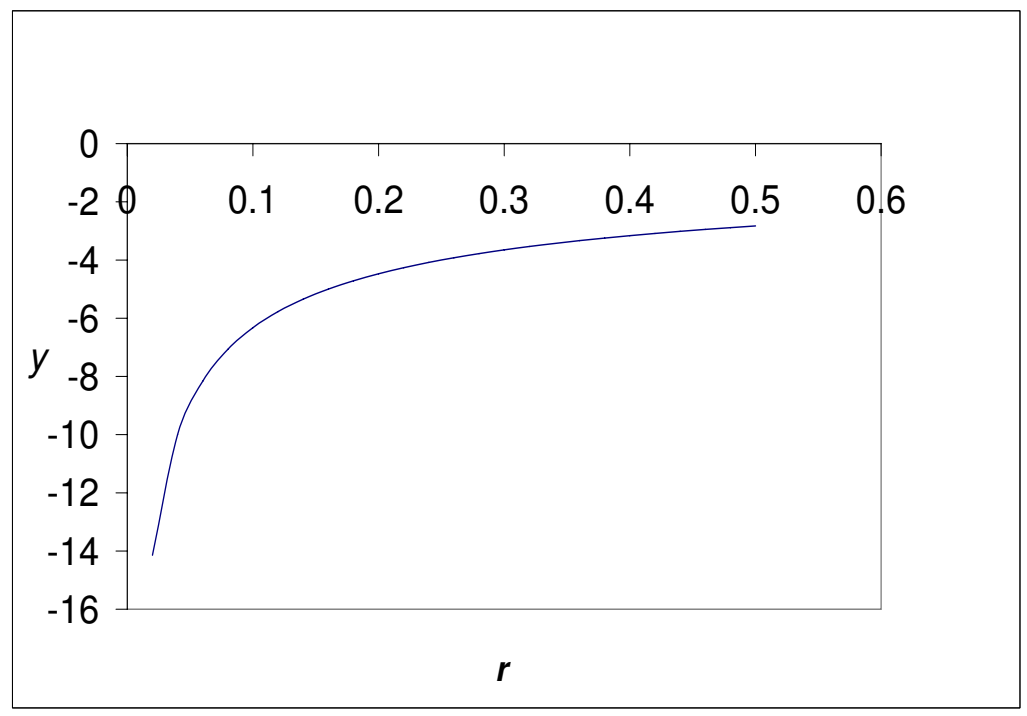

Figure 2: The plot for $y$ in (86) with respect to $r$ with $c=1$.

\section{Conclusions}

This paper studies two approximation methods, the moment approximation method and Kimmel's method, for solving general no-arbitrage bond price models. The two methods have been tested on two examples. We found that the modified formula of the moment approximate method can work very well if the current spot interest rate, as a determinant of the bond price, is not far from its long term equilibrium level in both cases. However, if the current spot is at some extreme level, there is a large error therefore the moment approximation method will fail in this case. We also found the nonlinearity considered in this paper increases approximation errors. Kimmel's method performs well in the special case based on the special transformation proposed in Kimmel (2008b) regarding both time and state transformations. However, if it deviates from this specific transformation, the procedure based on only the time transformation 
does not provide an accurate approximation, nor does the second order approximation approach for the application of the CIR model. Kimmel's method cannot be applied to Goard's example because it cannot fit into the second order approximation approach. 


\section{$5 \quad$ Table and Figures}
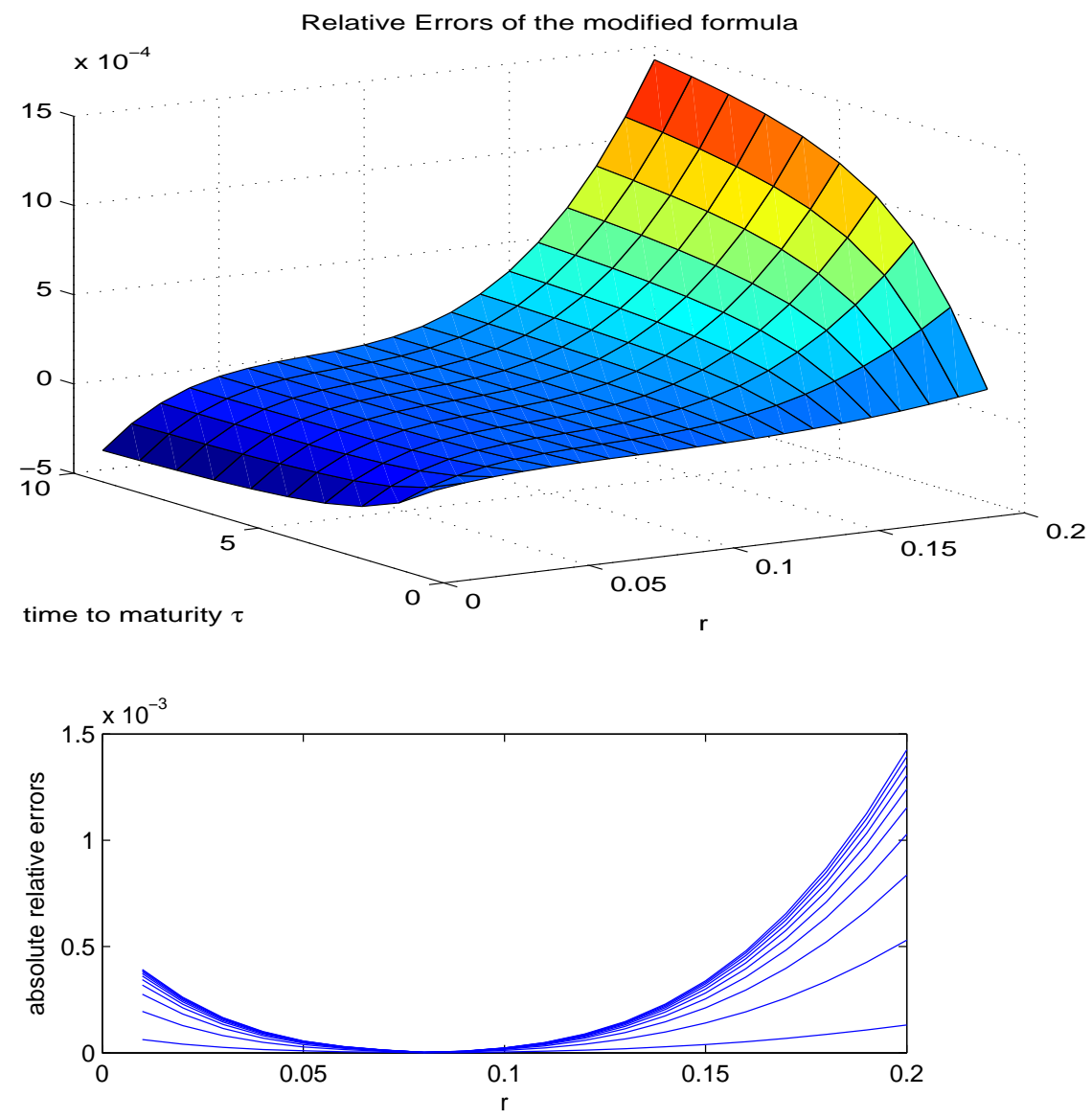

Figure 3: Relative Errors for the CIR Model $(\kappa=2.5)$

These panels illustrate the numerical behavior of the moment approximation method applied to the CIR model Eq. (24) with $\kappa=2.5, \theta=0.08, \sigma=0.15$. The first panel depicts the relative errors using the modified formula given in Eq. (13 for different $r_{t}$ levels and times to maturity. The second panel gives the relative errors against the levels $r_{t}$ for different times to maturity one year, two years,..., up to ten years (from bottom to top). The shaded area in the third panel gives $(r, \tau)$ combination where the relative error of the amended Shoji's formula is smaller than $1 \%$. The formula performs well around the equilibrium level of $r=0.08$. 

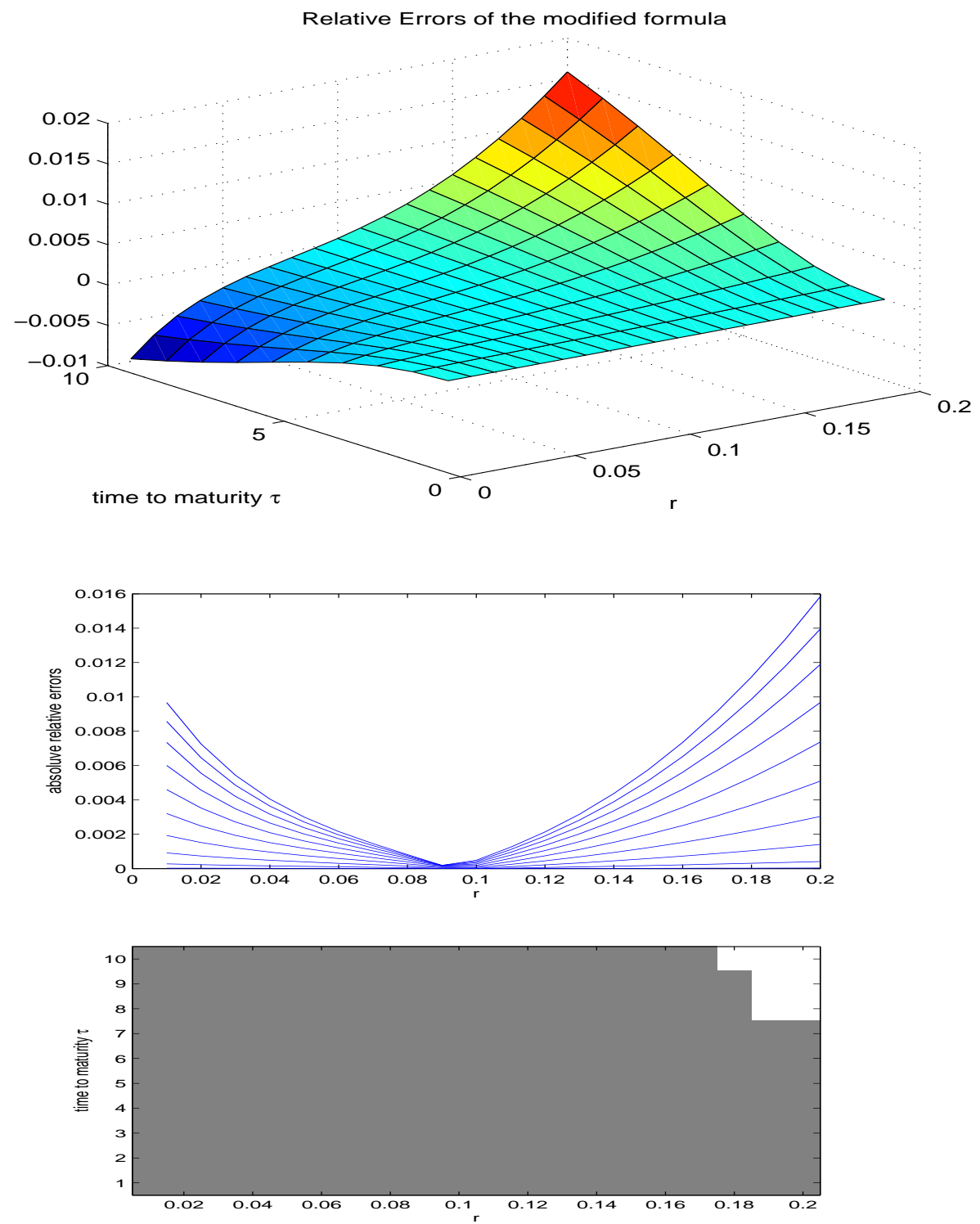

Figure 4: Relative Errors for the CIR Model ( $\kappa=0.5$ slow meareversion) These panels illustrate the numerical behavior of the moment approximation method applied to the CIR model Eq. (24) with $\kappa=0.5, \theta=0.08, \sigma=0.15$. The first panel depicts the relative errors using the modified formula given in Eq. (13 for different $r_{t}$ levels and times to maturity. The second panel gives the relative errors against the levels $r_{t}$ for different times to maturity one year, two years,..., up to ten years (from bottom to top). The shaded area in the third panel gives $(r, \tau)$ combination where the relative error of the amended Shoji's formula is smaller than $1 \%$. The formula performs well around the equilibrium level of $r=0.08$. 

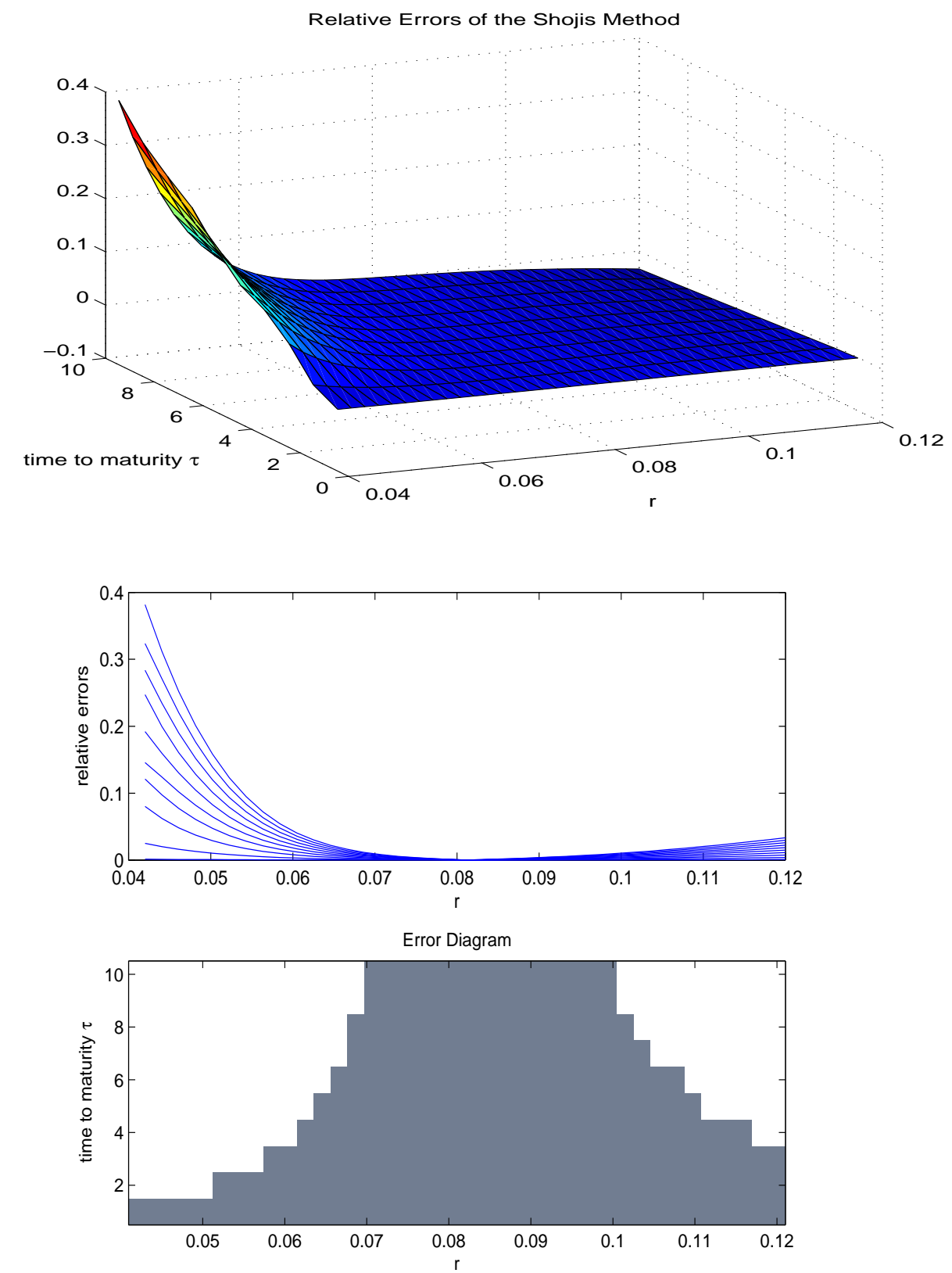

Figure 5: Relative Errors for Goard's Example $(q=30)$

The first panel depicts the relative errors using the modified formula given in Eq. (13 for different $r_{t}$ levels and times to maturity. The second panel shows the relative errors against the levels $r_{t}$ for different times to maturity one year, two years, ..., up to ten years (from bottom to top). The shaded area in the third panel gives $(r, \tau)$ combination where the relative error of the amended Shoji's formula is smaller than $1 \%$. The formula performs well around the equilibrium level of $r=0.08$. 

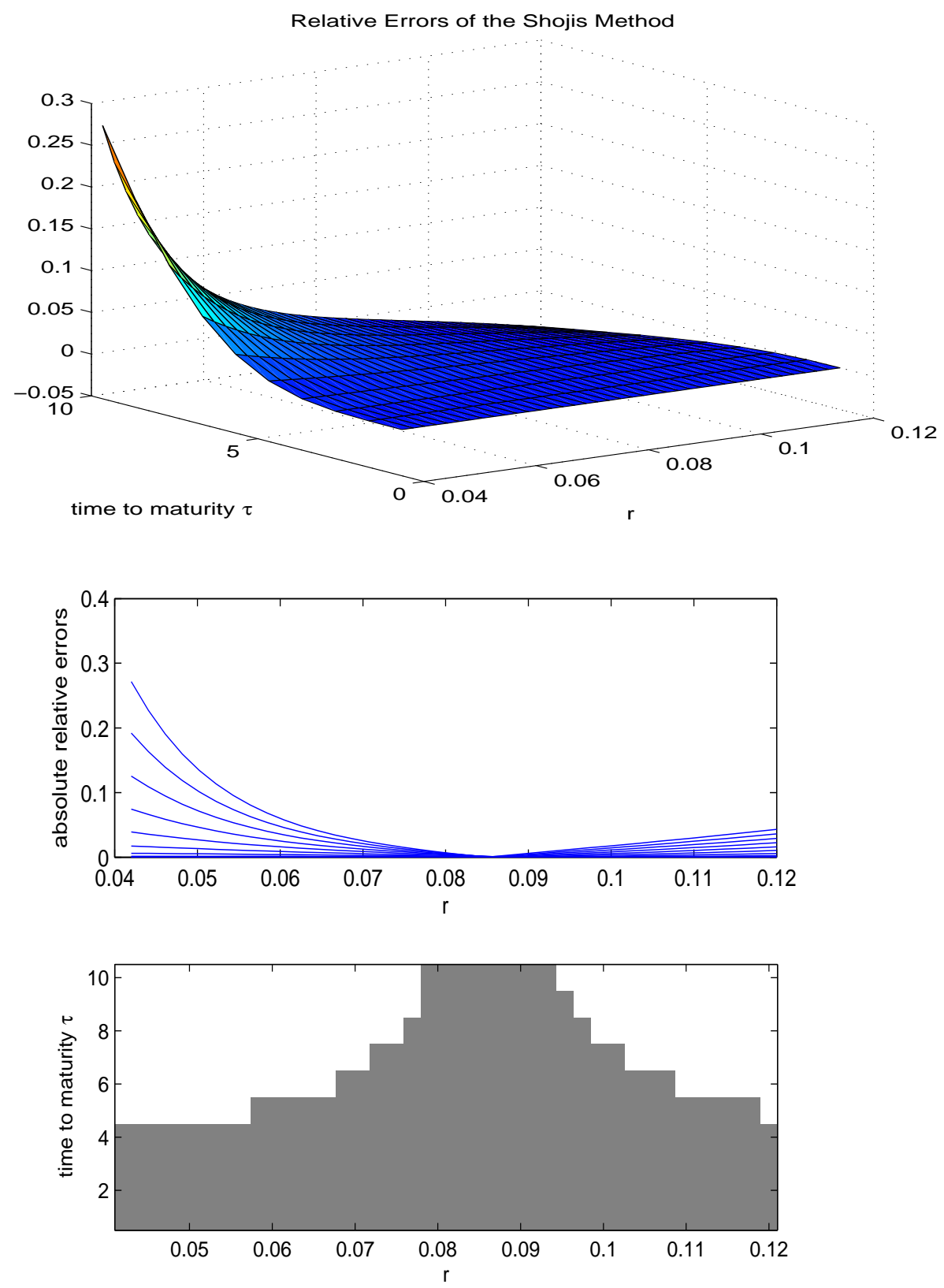

Figure 6: Relative Errors for Goard's Example ( $q=6.0$ slow mean reversion) The first panel depicts the relative errors using the modified formula given in Eq. (13 for different $r_{t}$ levels and times to maturity. The second panel shows the relative errors against the levels $r_{t}$ for different times to maturity one year, two years, ..., up to ten years (from bottom to top). The shaded area in the third panel gives $(r, \tau)$ combination where the relative error of the amended Shoji's formula is smaller than $1 \%$. The formula performs well around the equilibrium level of $r=0.08$. 


\begin{tabular}{rrrrr}
\hline T=1 & Bond Price & Rel Err(\%) & Yield & Rel Err(\%) \\
Exact & 0.9379129 & & 0.0640982 & \\
TS & 0.9379188 & $6.3288 \mathrm{E}-06$ & 0.0640919 & $-9.8736 \mathrm{E}-05$ \\
CH & 0.9379136 & $7.8474 \mathrm{E}-07$ & 0.0640974 & $-1.2243 \mathrm{E}-05$ \\
Kimmel $w(\xi, z)$ & 0.9379129 & $-5.0155 \mathrm{E}-06$ & 6.4098230 & $7.8247 \mathrm{E}-05$ \\
Kimmel $u(\xi, y)$ & 0.9383367 & $4.5181 \mathrm{E}-02$ & 6.3646468 & $-7.0472 \mathrm{E}-01$ \\
Kimmel App. & 0.9424313 & $4.8175 \mathrm{E}-01$ & 5.9292253 & $-7.4978 \mathrm{E}+00$ \\
\hline $\mathbf{T = 5}$ & Bond Price & Rel Err(\%) & Yield & Rel Err(\%) \\
Exact & 0.7003148 & & 0.0712451 & \\
TS & 0.7017311 & $2.0223 \mathrm{E}-03$ & 0.0708410 & $-5.6714 \mathrm{E}-03$ \\
CH & 0.7005387 & $3.1965 \mathrm{E}-04$ & 0.0711811 & $-8.9717 \mathrm{E}-04$ \\
Kimmel $w(\xi, z)$ & 0.7003131 & $-2.4301 \mathrm{E}-04$ & 7.1245553 & $6.8219 \mathrm{E}-04$ \\
Kimmel $u(\xi, y)$ & 0.7858766 & $1.2218 \mathrm{E}+01$ & 4.8191103 & $-3.2359 \mathrm{E}+01$ \\
Kimmel App. & 0.7864343 & $1.2298 \mathrm{E}+01$ & 4.8049223 & $-3.2558 \mathrm{E}+01$ \\
\hline $\mathbf{T = 1 0}$ & Bond Price & Rel Err(\%) & Yield & Rel Err(\%) \\
Exact & 0.4780730 & & 0.0737992 & \\
TS & 0.4853738 & $1.5271 \mathrm{E}-02$ & 0.0722836 & $-2.0536 \mathrm{E}-02$ \\
CH & 0.4788345 & $1.5927 \mathrm{E}-03$ & 0.0736400 & $-2.1564 \mathrm{E}-03$ \\
Kimmel $w(\xi, z)$ & 0.4780715 & $-3.2971 \mathrm{E}-04$ & 7.3799506 & $4.4677 \mathrm{E}-04$ \\
Kimmel $u(\xi, y)$ & 0.7581700 & $5.8589 \mathrm{E}+01$ & 2.7684772 & $-6.2486 \mathrm{E}+01$ \\
Kimmel App. & 0.6532927 & $3.6651 \mathrm{E}+01$ & 4.2573006 & $-4.2312 \mathrm{E}+01$ \\
\hline & & & & \\
\hline
\end{tabular}

Table 3: Numerical results for the moment approximation method and Kimmel's method in the case of the CIR model. Parameter values: $\kappa=0.5, \theta=0.08$, $\sigma=0.15, r_{0}=0.06, \bar{y}_{0}=2 \sqrt{r_{0}} / \sigma$ and the power series terms up to $N=$ 4. The results of Takamizawa/Shoji's formula (TS), Chiarella/Hsiao's formula $(\mathrm{CH})$, "Kimmel $w(\xi, z)$ ", "Kimmel $u(\xi, y)$ " and "Kimmel App." are based on Subsections 2.1.1, 2.1.2, 3.2.1, 3.2.2 and 3.2.3 respectively.

\section{Appendix}

Proof of Property 5

For notational convenience we denote $A:=A_{s}^{a d}, B:=B_{s}^{a d}, \Psi(t)=\Psi_{s}^{a d}(t)$ and $\Phi(t)=\Phi_{s}^{a d}(t)$. Recall $\Psi(t)$ satisfies (10) and $\Phi(t)$ satisfies $(12)$.

Define a new function $w_{s}(t)$ satisfying

$$
w(t)=A \int_{s}^{t} w(u) d u+\left(B+R_{\max }\right) \cdot(t-s) .
$$

Define a difference process $\hat{w}(t):=w(t)-\Phi(t)$. From the dynamics (12) and (89) we derive the dynamics of $\hat{w}(t)$ as 
$\hat{w}(t)=A \int_{s}^{t}(w(u)-\Phi(u)) d u+R_{\max }(t-s)=A \int_{s}^{t} \hat{w}(u) d t+R_{\max }(t-s)$.

Now consider another difference process $\hat{\Psi}(t):=|\Psi(t)-\Phi(t)|$. Similarly the dynamics are given by

$$
\begin{aligned}
\hat{\Psi}(t)=|\Psi(t)-\Phi(t)| & =\left|A \int_{s}^{t}(\Psi(u)-\Phi(u)) d u+R^{a d}(t)\right| \\
& \leq A \int_{s}^{t}|\Psi(u)-\Phi(u)| d u+\left|R^{a d}(t)\right| \\
& \leq A \int_{s}^{t} \hat{\Psi}(u) d u+R_{\max }(t-s) .
\end{aligned}
$$

The initial conditions of the two difference processes are $\hat{w}(s)=\hat{\Psi}(s)=0$. Use the Growwall's Lemma on p.284 Walter (1996) we have

$$
\hat{\Psi}(t) \leq \hat{w}(t)=A^{-1}\left(e^{A(t-s)}-I\right) R_{\max } .
$$

\section{References}

Agn, D., R. Dittmar and A. Gallant (2002), 'Quadratic term sturcture models: Theory and evidence', Review of Financial Studies 15, 243-288.

Chiarella, C. and Hsiao, C.-Y. (2010), Solving nonlinear term structure models using moment approximation methods with an empirical study on the U.S. bond market. working paper in preparation.

Colton, D. (1979), 'The approximation of solutions to the backwards heat equation in a nonhomogeneous medium', Journal of Mathematical Analysis and Applications 72, 418-429.

Goard, J. (2000), 'New solutions to the bond-pricing equation via Lie's classical method', Mathematical and Computing Modelling 32, 299-313.

Kimmel, R. (2008a), 'Changing times: Asset pricing and conditional moments under non-affine diffusions', Working paper, Fisher College of Business, The Ohio State University .

Kimmel, R. (2008b), 'Changing times: The pricing problem in non-linear models', Working paper, Department of Finance, The Ohio State University 
Shoji, I. (2002), 'Approximation of conditional moments of diffusion processes', International Journal of Computational and Numerical Analysis and Applications 1(2), 163-190.

Takamizawa, H. and Shoji, I. (2009), 'Modeling the term structure of interest rates with general diffusion processes: a moment approximation approach', Journal of Economic Dynamics and Control 33, 65-77.

Walter, W. (1996), Gewönlich Differentialgleichungen (Ordinary Differential Equations), 6th edn, Springer. 